\title{
Impact of a Rapid Point of Care Test for Influenza on Guideline Consistent Care and Antibiotic Use
}

\author{
Ariella Perry Dale, PhD, MPH, Mark Ebell, MD, MS, Brian McKay, MPH, \\ Andreas Handel, PhD, Ronald Forehand, MD, and Kevin Dobbin, PhD
}

Background: Rapid influenza diagnostic tests that detect the presence of viral antigens are currently used throughout the United States but have poor sensitivity. The objective of this study was to identify if the use of a new highly accurate rapid point of care test would significantly increase the likelihood of guideline consistent care.

Methods: We prospectively recruited 300 students at a university health clinic who presented with cough and 1 influenza-like illness symptom between December 2016 and February 2017 to receive care guided by a rapid polymerase chain reaction (PCR) test. of the 300 patients receiving the PCR test, 264 had complete medical records and were compared to 771 who received usual care. We used a logistic regression model to identify whether PCR guided care was associated with guideline consistent care, based on the appropriate use of oseltamivir and antibiotics. We also assessed whether PCR guided care decreased the likelihood of return visits within 2 weeks by patients.

Results: Logistic regression revealed that the odds of receiving guideline supported care did not significantly increase for patients who received PCR guided care (adjusted odds ratio [aOR], 1.24; 95\% CI, 0.83-1.88). It significantly decreased the likelihood of an antibiotic prescription (a0R, 0.61; 95\% CI, 0.40-0.94), increased the likelihood of receiving oseltamivir (a0R, 1.57; 95\% CI, 1.09-2.28), and decreased the likelihood of return visit within 2 weeks (aOR, $0.19 ; 95 \% \mathrm{CI}, 0.04-0.81$ ).

Conclusions: The use of a rapid PCR test did not significantly improve the likelihood of guideline consistent care. However, independent of test outcome, patients who received the test were more likely to receive an antiviral and less likely to receive an antibiotic or have a return visit within 2 weeks.

(J Am Board Fam Med 2019;32:226-233.)

Keywords: Antibiotics, Antiviral Agents, Diagnostic Tests, Influenza, Point of Care Testing, Polymerase Chain Reaction

Influenza remains a large burden in the United States. Beginning in 2009, the Centers for Disease Control and Prevention (CDC) began tracking the number of outpatient medical visits attributed to influenza. At the peak of the 2017 to 2018 season,

This article was externally peer reviewed.

Submitted 28 June 2018; revised 1 November 2018; accepted 7 November 2018.

From Department of Epidemiology and Biostatistics, University of Georgia, Athens (APD, ME, BM, AH, KD); Center for Ecology of Infectious Diseases, University of Georgia, Athens (AH); University Health Center, University of Georgia, Athens (RF).

Funding: This study was funded by Roche Diagnostics.

Conflict of interest: none declared.

Corresponding author: Ariella Perry Dale, PhD, MPH, Department of Epidemiology and Biostatistics, University of Georgia, Athens, GA 30602 (E-mail: aperrydale@ gmail.com). approximately $7.5 \%$ of outpatient office visits were attributed to influenza. ${ }^{1}$ The current treatment of influenza is primarily supportive with the selective use of a neuraminidase inhibitor, primarily oseltamivir. ${ }^{4}$ In patients with influenza and a low risk for bacterial infections, antibiotics are not guideline consistent. $^{5}$ The use of antibiotics remains common for many viral acute respiratory infections, emphasizing the need for clinician and patient education regarding their ineffectiveness for these pathogens. ${ }^{4}$ One reason for the overprescription of antibiotics is the overlapping signs and symptoms between many respiratory infections. Clinicians also cite concern of a bacterial pneumonia infection as one of the leading reasons for prescribing an antibiotic in patients with influenza or lower respiratory tract infection. ${ }^{7}$ 
Figure 1. Pragmatic trial diagram of recruitment of polymerase chain reaction (PCR) guided and usual care patients across 3 clinics. EHR, electronic health record.

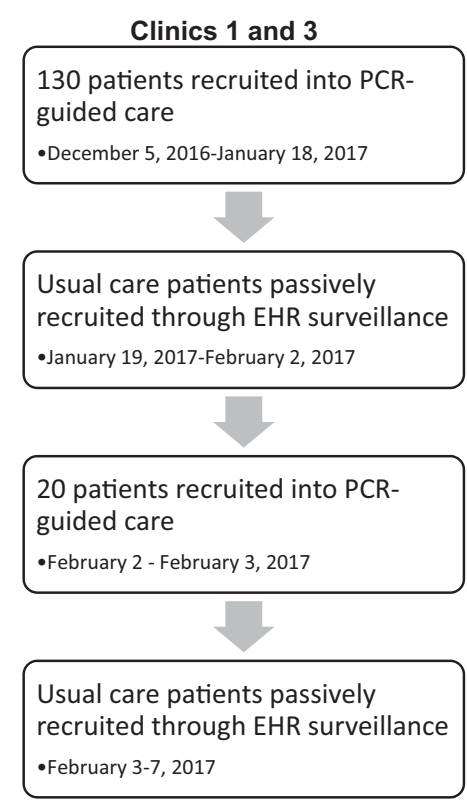

Another reason for the overprescription of antibiotics is low confidence in rapid influenza diagnostic tests, which have poor sensitivity. ${ }^{8}$ A metaanalysis of 159 studies showed a pooled sensitivity of $62.3 \%$ (95\% CI, $57.9 \%-66.6 \%){ }^{7}$ Polymerase chain reaction (PCR) tests are the standard in influenza diagnosis but until recently have taken 24 to 36 hours to yield results. ${ }^{8}$ The recent introduction of a rapid point of care PCR test for influenza A and $\mathrm{B}$ combines the timeliness of the current rapid flu tests with the increased sensitivity and specificity of traditional PCR. ${ }^{9}$ The rapid point of care PCR test takes approximately 20 minutes to complete. The result is a qualitative PCR result for the presence or absence of influenza A or influenza B. This test is $99 \%$ to $100 \%$ sensitive and specific for influenza A and B when compared to a reference laboratory PCR assay. ${ }^{9}$ We hypothesize that this will increase guideline consistent prescribing of antivirals and antibiotics in patients with acute respiratory infections and decrease the likelihood of return visits within 2 weeks.

\section{Methods}

This was a prospective, quasiexperimental, nonrandomized comparison of PCR guided care with usual care in patients presenting with influenza-like illness (ILI). Usual care could include the use of the Quidel Quickvue Strep A rapid diagnostic test
Clinic 2

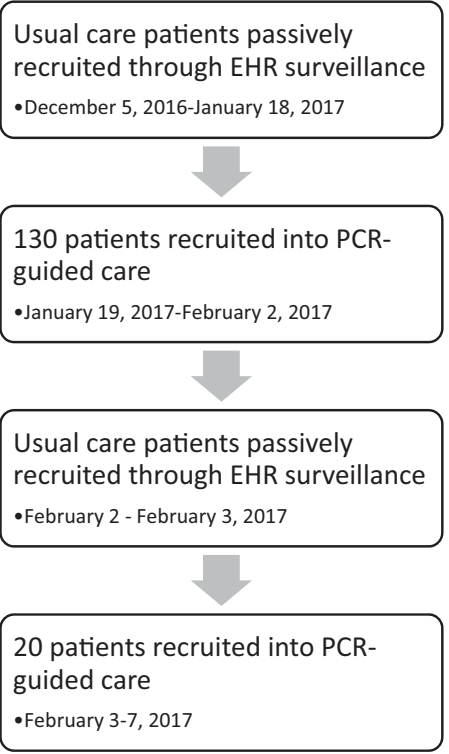

(standard at the time of this study). Previous studies demonstrate that the sensitivity of this rapid diagnostic test can be as low as $58 \% .^{9}$ Due to logistic constraints imposed by the clinical site, randomization was not feasible. Clinicians were told that the goal of the study was to study influenza diagnosis and were not told that it was explicitly to study antibiotic or antiviral use to avoid a Hawthorne effect. During periods where the PCR test was in use in a clinic, the test result report clearly stated that the result was from a novel, highly accurate PCR test within the electronic health record. The majority of signs and symptoms were captured by Boolean or check boxes.

\section{Population Studied}

There were 2 study groups: PCR guided and usual care. Recruitment for both groups took place from December 2016 to February 2017 at 3 primary care clinics within the University Health Center at the University of Georgia, as shown in Figure 1. Student's visits with a clinician are free; diagnostic testing and prescriptions are not covered by the health fee. In the PCR guided group, patients with an acute respiratory infection were identified in the clinic schedule and were enrolled if they had a cough and at least 1 ILI symptom and presented to the clinic within 7 days of symptom onset. Patients in the usual care group were identified based on 


\begin{tabular}{llr}
\hline & & \multicolumn{2}{c}{ Guideline Treatment } \\
\cline { 3 - 3 } Diagnosis & Oseltamivir & Antibiotics \\
\hline $\begin{array}{l}\text { Influenza positive (PCR confirmed or final clinical diagnosis) } \\
\begin{array}{l}\text { No influenza, but high risk for pneumonia or other bacterial } \\
\text { infection diagnosis }\end{array}\end{array}$ & $\begin{array}{l}\text { Consistent }(<48 \text { hours onset) } \\
\text { No influenza, low risk for pneumonia and no bacterial } \\
\text { infection diagnosis }\end{array}$ & Inconstent \\
\hline
\end{tabular}

PCR, polymerase chain reaction.

their responses to required fields in the electronic health record regarding respiratory symptoms and were similarly included in the study if they had a cough and at least 1 other symptom of ILI within 7 days of symptom onset. Recruitment for the PCR and usual care group was alternated between clinics during 2 time periods, so that patients from all 3 clinics could be in either study group. For example, patients were recruited for the PCR guided care arm in clinic 2, whereas patients in clinics 1 and 3 meeting the same inclusion criteria during that time were included in the usual care arm. The recruitment algorithm then swapped; patients from clinics 1 and 3 were recruited for the PCR guided care arm, wherease patients in clinic 2 were included in the usual care arm. Patients were considered not enrolled in the study if they presented to 1 of the clinics during enrollment for PCR guided care but either refused participation or the research assistant was not available.

\section{Intervention and Outcome Variable Definitions}

Our intervention was the type of care a patient received (PCR guided Vs usual), and our outcome was guideline consistent care (consistent Vs inconsistent). A patient was considered influenza positive if they had a positive rapid point of care PCR test or a final clinical diagnosis of influenza. The definition of influenza positive included both the PCRbased and final clinician diagnosis because not all patients in the usual care group received a point of care test. A patient was considered influenza negative if they had a negative rapid point of care PCR test and no final clinical diagnosis of influenza.

Table 1 defines guideline consistent and guideline inconsistent treatment depending on the diagnosis. Oseltamivir is only guideline consistent when administered within 48 hours of symptom onset in patients with influenza confirmed by PCR or clini- cian diagnosis per the Food and Drug Administration. ${ }^{10}$ Antibiotics are guideline consistent in patients with a high risk of pneumonia or a final clinical diagnosis of a bacterial infection. Patients with a final diagnosis of acute otitis media or acute bacterial rhinosinusitis were excluded because antibiotic prescriptions are recommended for selected patients based on US treatment guidelines. ${ }^{11,12} \mathrm{~Pa}$ tients who are diagnosed with influenza more than 48 hours after symptom onset, that do not have influenza, are at low risk for pneumonia, and do not have a bacterial infection as a clinical diagnosis should not receive oseltamivir or antibiotics.

\section{Analysis}

All analyses were performed in $\mathrm{R}$ version 3.3.3. We used $\chi^{2}$ testing to determine any preliminary differences between PCR guided and usual care groups. We created 4 logistic regression models by using a manual forward addition strategy based on the Aikake Information Criteria (AIC) to identify the association between PCR guided care and our outcomes of interest. These outcomes include guideline consistent care, antibiotic prescription, antiviral prescription, and return visit within 2 weeks. Each model was adjusted for signs, symptoms, and clinic assignment. This technique involves beginning with the crude model and creating a model that adds characteristics. The AIC for each model was recorded and compared to the previous model. The change in AIC between the 2 models was assessed, and the model with the largest decrease in AIC was selected as the better fit. ${ }^{13}$ This process was continued until the AIC change was less than or equal to $3 .^{13}$

\section{Results}

During the study period, 3,095 patients with a chief respiratory complaint were seen in the 3 University 
Table 2. Signs, Symptoms, and Clinic Assignments for polymerase chain reaction (PCR) Guided Care and Usual

Care

\begin{tabular}{|c|c|c|c|c|c|}
\hline \multirow[b]{2}{*}{ Characteristic } & \multicolumn{2}{|c|}{$\begin{array}{l}\text { PCR Guided Care } \\
\quad(\mathrm{n}=264)\end{array}$} & \multicolumn{2}{|c|}{$\begin{array}{l}\text { Usual Care } \\
(\mathrm{n}=771)\end{array}$} & \multirow{2}{*}{$\begin{array}{l}P \text { value PCR Vs } \\
\text { Usual Care }\end{array}$} \\
\hline & $\mathrm{N}$ & Frequency (\%) & $\mathrm{N}$ & Frequency (\%) & \\
\hline Cough & 264 & 100.0 & 771 & 100.0 & \\
\hline Sore throat & 253 & 95.8 & 726 & 94.2 & .30 \\
\hline Nasal discharge & 243 & 92.1 & 670 & 86.9 & .03 \\
\hline Headache & 234 & 88.6 & 620 & 80.4 & $<.01$ \\
\hline Chills & 229 & 86.7 & 556 & 72.1 & $<.01$ \\
\hline Fever & 192 & 72.7 & 428 & 55.5 & $<.01$ \\
\hline Congestion & 192 & 72.7 & 534 & 69.3 & .29 \\
\hline Myalgia & 179 & 67.8 & 399 & 51.8 & $<.01$ \\
\hline Pharynx erythema & 154 & 58.3 & 371 & 48.1 & $<.01$ \\
\hline Nausea & 55 & 20.8 & 121 & 15.7 & .06 \\
\hline Enlarged tonsils & 29 & 11.0 & 81 & 10.5 & .83 \\
\hline Vomit & 27 & 10.2 & 60 & 7.8 & .22 \\
\hline Unclear lungs & 27 & 10.2 & 53 & 6.9 & .08 \\
\hline Diarrhea & 23 & 8.7 & 96 & 12.5 & .10 \\
\hline Pharynx exudate & 12 & 4.6 & 25 & 3.2 & .33 \\
\hline Tonsillar exudate & 7 & 2.7 & 35 & 4.5 & .18 \\
\hline Rales & 5 & 1.9 & 29 & 3.8 & .14 \\
\hline Lung distress & 0 & 0.0 & 3 & 0.4 & .31 \\
\hline Clinic 1 & 75 & 28.4 & 238 & 30.9 & $<.01$ \\
\hline Clinic 2 & 134 & 50.8 & 270 & 35.0 & \\
\hline Clinic 3 & 55 & 20.8 & 263 & 34.1 & \\
\hline Duration $\leq 2$ days & 131 & 49.6 & 296 & 38.4 & $<.01$ \\
\hline$>2$ days & 133 & 50.4 & 475 & 61.6 & \\
\hline Day of visit Friday & 59 & 22.4 & 205 & 18.7 & .20 \\
\hline Day of visit other weekday & 205 & 77.6 & 627 & 81.3 & \\
\hline
\end{tabular}

Bold text indicates statistically significant values.

PCR, polymerase chain reaction.

Health Center clinics. A total of 300 patients were enrolled to receive care guided by the rapid PCR test. Twelve of these patients were excluded from our final analysis due to an invalid PCR test result. An invalid PCR test result indicates that the presence or absence of influenza cannot be determined; a new sample should be obtained. Seven patients who received an invalid result agreed to be tested again, although several of these received another invalid result $(\mathrm{n}=5)$. Seven patients were missing all sign and symptom data and, therefore, were excluded from our analysis. The visit notes for these 7 patients were reviewed but did not contain the information necessary to be included in the analysis. Some patients had both an invalid PCR result and no sign or symptom data. Finally, we excluded 16 patients for having no reported cough. There are 2 explanations for this phenomenon. In a few cases, our study recruitment staff erroneously included persons who had 2 suspected influenza symptoms instead of cough plus 1 suspected influenza symptom. Second, patients seeking enrollment into the study may have reported a cough verbally to the study enrollment staff but not to their clinician.

Therefore, 264 patients were included in the final PCR guided care group for analysis. Patients were designated as receiving usual care if they presented with a respiratory infection and met the inclusion criteria used by the PCR guided care group but presented to a clinic that was not currently enrolling patients to receive the PCR test at the time of visit. In the 3 clinics, 771 patients presented with the same inclusion criteria and received usual care. Of these patients, 234 (30.4\%) received a standard rapid influenza test (not PCR). 
Table 2 presents the symptoms, signs, and location breakdown for each care group. Clinic 1 had 75 PCR-tested patients, clinic 2 had 134 PCR-tested patients, and clinic 3 had 55 PCR-tested patients.

We compared our final entire data set (PCR guided care group and usual care group, $\mathrm{n}=1035$ ) to the patients who met inclusion criteria in the clinic during PCR recruitment but were unenrolled $(\mathrm{n}=518)$. These patients were not included in the final data set.

\section{Description of Guideline Consistent or Inconsistent Care}

Overall, 193 of the 1,035 patients in both groups received guideline inconsistent care $(18.7 \%)$. Of 312 patients given oseltamivir, $10 \mathrm{did}$ not have a PCR-positive test or a clinical diagnosis of influenza. Of 191 patients given an antibiotic, only 24 were at high risk of pneumonia or were given a diagnosis consistent with bacterial infection. Of all patients who received oseltamivir, $27.5 \%$ had a duration of symptoms greater than 48 hours. In the PCR guided care group, 27 of 122 patients with a negative PCR test $(22.1 \%)$ were still given a final clinical diagnosis of influenza, and 5 of those 27 patients received oseltamivir. In the usual care group, 537 of 772 (69.6\%) patients did not receive a rapid influenza test, of whom $66(21.2 \%)$ received an oseltamivir prescription based on clinical diag-

Table 3. Final Adjusted Model* for the Association between polymerase chain reaction (PCR) Guided Care and Guideline Consistent Care

\begin{tabular}{lcc}
\hline Sign/Symptom & $\begin{array}{c}\text { Estimate } \\
(\beta \text { coefficient })\end{array}$ & aOR (95\% CI) \\
\hline PCR guided care & 0.22 & $1.24(0.83-1.88)$ \\
Tonsillar exudate & -1.76 & $0.17(0.07-0.39)$ \\
Clinic 2 & -0.28 & $0.76(0.48-1.18)$ \\
Clinic 3 & 0.58 & $1.79(1.09-2.93)$ \\
Myalgia & 0.85 & $2.35(1.61-3.42)$ \\
Pharynx erythema & -0.60 & $0.55(0.37-0.82)$ \\
Pharynx exudate & -1.25 & $0.29(0.13-0.63)$ \\
Rales & -0.98 & $0.38(0.17-0.81)$ \\
Enlarged tonsils & -0.80 & $0.45(0.26-0.78)$ \\
Duration of symptoms & 0.55 & $1.74(1.17-2.57)$ \\
$\quad(\leq 2$ days Vs $>2$ days $)$ & & \\
Unclear lungs & -0.78 & $0.46(0.26-0.82)$ \\
Intercept & 1.49 & \\
\hline
\end{tabular}

${ }^{*} \mathrm{H}-\mathrm{L} \chi^{2}$ statistic, 10.9; $P=.21$; AUROCC, 0.77 .

aOR, adjusted odds ratio; CI, confidential interval; PCR, polymerase chain reaction.
Table 4. Final Adjusted Model*† for the Likelihood of Antiviral Prescription

\begin{tabular}{lcc}
\hline Sign/Symptom & $\begin{array}{c}\text { Estimate } \\
(\beta \text { coefficient })\end{array}$ & aOR $(95 \%$ CI $)$ \\
\hline PCR guided care ${ }^{\dagger}$ & 0.46 & $1.57(1.09-2.28)$ \\
Myalgia & 1.30 & $3.65(2.35-5.68)$ \\
$\begin{array}{l}\text { Duration of symptoms } \\
\quad(\leq 2 \text { days Vs }>2 \text { days })\end{array}$ & 1.77 & $5.89(4.18-8.28)$ \\
Fever & 0.93 & $2.52(1.57-4.05)$ \\
Tonsillar exudate & -1.67 & $0.19(0.05-0.77)$ \\
Unclear lungs & 1.05 & $2.86(1.58-5.17)$ \\
Clinic 2 & 0.13 & $1.13(0.75-1.72)$ \\
Clinic 3 & 0.74 & $2.09(1.34-3.25)$ \\
Nasal discharge & 0.80 & $2.24(1.19-4.19)$ \\
Chills & 0.87 & $2.38(1.25-4.53)$ \\
Enlarged tonsils & -0.71 & $0.49(0.25-0.95)$ \\
Intercept & -5.13 & \\
\hline
\end{tabular}

${ }^{*}$ Hosmer-Lemeshow $\chi^{2}$ statistic, 3.16; $\mathrm{df}=10 ; P=.92$; AUROCC, 0.86 .

${ }^{\dagger}$ Unadjusted OR, 2.10 (1.57-2.82).

aOR, adjusted odds ratio; CI, confidential interval; PCR, polymerase chain reaction.

nosis. Finally, the PCR guided care group had no significant difference in the odds of receiving guideline consistent care compared to the usual care group in univariate logistic regression analysis $(83.7 \%$ Vs $80.5 \%$, respectively; $P=.25$; OR, 1.24 ; 95\% CI, 0.86-1.80).

\section{Effect of PCR Guided Care on Guideline Consistent Care}

The final adjusted logistic regression model is shown in Table 3. The association between use of the PCR test and a greater likelihood of guideline consistent care was not statistically significant (adjusted odds ratio [aOR], 1.24; 95\% CI, 0.82-1.88), with a trend favoring guideline consistent care in the PCR guided care group. The Hosmer-Lemeshow $\chi^{2}$ statistic was 10.9 with a $P$ value of 0.21 , indicating a good fit, and the area under the receiver operating characteristic curve (AUROCC) was 0.77 .

\section{Effect of PCR Guided Care on Prescription of Antibiotics or Antivirals and Return Visit}

We also conducted post hoc multivariate analyses to study the association between PCR guided versus usual care on the likelihood of antiviral prescription (Table 4), antibiotic prescription (Table 5 ), and the likelihood of a return visit in the 2 weeks 
Table 5. Final Adjusted Model ${ }^{* \dagger}$ for the Likelihood of Antibiotic Prescription

\begin{tabular}{lrc}
\hline Sign/Symptom & $\begin{array}{c}\text { Estimate } \\
(\beta \text { coefficient })\end{array}$ & aOR (95\% CI) \\
\hline PCR guided care $^{\dagger}$ & -0.31 & $0.61(0.40-0.94)$ \\
Tonsil exudate & 1.63 & $5.10(2.14-12.1)$ \\
Pharynx erythema & 0.96 & $2.60(1.73-3.93)$ \\
Pharynx exudate & 1.78 & $5.94(2.56-13.8)$ \\
Duration of symptoms & -0.73 & $0.48(0.33-0.71)$ \\
$\quad(\leq 2$ days vs $>2$ days $)$ & & \\
Sore throat & 0.60 & $1.83(0.63-5.26)$ \\
Enlarged tonsils & 0.89 & $2.43(1.42-4.16)$ \\
Unclear lungs & 0.79 & $2.21(1.26-3.88)$ \\
Nasal discharge & -0.65 & $0.52(0.31-0.87)$ \\
Clinic 2 & 0.09 & $1.10(0.70-1.73)$ \\
Clinic 3 & -0.44 & $0.64(0.39-0.05)$ \\
Intercept & -1.99 & \\
\hline
\end{tabular}

${ }^{*}$ Hosmer-Lemeshow $\chi^{2}$ statistic, 6.32; $\mathrm{df}=8 ; P=.61$; AUROCC, 0.79 .

${ }^{\dagger}$ Unadjusted OR, $0.71(0.48-1.04)$.

aOR, adjusted odds ratio; CI, confidential interval; PCR, polymerase chain reaction.

following the initial appointment (Table 6) by using manual AIC logistic regression model building. Patients who received PCR guided care were significantly more likely to receive an oseltamivir prescription (aOR, 1.58; 95\% CI, 1.09-2.28) and significantly less likely to receive an antibiotic prescription (aOR, 0.61; 95\% CI, 0.40-0.94). Of PCR guided care patients, $42 \%$ received an oseltamivir prescription and $15 \%$ received an antibiotic prescription (in usual care $26 \%$ and $19.7 \%$, respectively). In light of these findings, we conducted a brief analysis of the likelihood of a receiving a guideline consistent antiviral prescription. Patients receiving PCR guided care were significantly more likely to receive a guideline consistent antiviral pre-

Table 6. Final Adjusted Model* for the Likelihood of Return Visit

\begin{tabular}{|c|c|c|}
\hline Sign/Symptom & $\begin{array}{c}\text { Estimate } \\
(\beta \text { coefficient })\end{array}$ & aOR (95\% CI) \\
\hline PCR guided care & -1.66 & $0.19(0.04-0.81)$ \\
\hline $\begin{array}{l}\text { Day of visit (Friday Vs } \\
\text { other weekdays) }\end{array}$ & 1.04 & $2.83(1.29-6.19)$ \\
\hline Pharynx erythema & 0.97 & $2.65(1.15-6.10)$ \\
\hline Intercept & -4.22 & . \\
\hline
\end{tabular}

${ }^{*}$ Hosmer-Lemeshow $\chi^{2}$ statistic, 2.56; $P=.63$; AUROCC, 0.70. aOR, adjusted odds ratio; CI, confidential interval; PCR, polymerase chain reaction.
Table 7. Final Adjusted Model ${ }^{* \dagger}$ for the Likelihood of Guideline Consistent Antiviral Prescription

\begin{tabular}{lcc}
\hline Sign/Symptom & $\begin{array}{c}\text { Estimate } \\
(\beta \text { coefficient })\end{array}$ & aOR (95\% CI) \\
\hline PCR guided care & 0.53 & $1.71(1.18-2.46)$ \\
$\begin{array}{l}\text { Duration of symptoms } \\
\quad(\leq 2 \text { days vs }>2 \text { days })\end{array}$ & 1.76 & $5.82(4.14-8.19)$ \\
Myalgia & 1.29 & $3.62(2.32-5.63)$ \\
Fever & 0.88 & $2.41(1.50-3.89)$ \\
Enlarged tonsils & -1.04 & $0.35(0.20-0.64)$ \\
Clinic 2 & 0.12 & $1.13(0.75-1.71)$ \\
Clinic 3 & 0.77 & $2.15(1.39-3.35)$ \\
Unclear lungs & 0.82 & $2.28(1.27-4.08)$ \\
Nasal discharge & 0.79 & $2.20(1.18-4.10)$ \\
Chills & 0.80 & $2.20(1.17-4.24)$ \\
Intercept & -5.10 & \\
\hline
\end{tabular}

*Hosmer-Lemeshow $\chi^{2}$ statistic, 5.67; $P=.68$; AUROCC, 0.86 . aOR, adjusted odds ratio; CI, confidential interval; PCR, polymerase chain reaction.

scription (aOR, 1.71; 95\% CI, 1.18-2.46; Table 7). In addition, the odds of a return visit for patients who received PCR guided care were significantly lower than for patients who received usual care (aOR, 0.19; 95\% CI, 0.04-0.81).

\section{Discussion}

PCR guided care was associated with a small but nonsignificant increase in the percentage of patients that received guideline consistent care. In addition, patients receiving PCR guided care were significantly more likely to receive a prescription for oseltamivir but were significantly less likely to receive an antibiotic prescription. Finally, patients were also significantly less likely to return for a second visit within 2 weeks if they received PCR guided care. This reduction in antibiotic use and return visits is especially notable because patients in the PCR guided care group were more likely to have symptoms of influenza and had more symptoms than those in the usual care group. Thus, the use of a highly accurate PCR test has the potential to help reduce the inappropriate use of antibiotics and the need for return visits. We hypothesize that this association is due to a greater diagnostic confidence with the highly accurate PCR test. On the other hand, the greater sensitivity of the PCR test resulted in more patients being correctly diagnosed with influenza, leading to more prescribing of oseltamivir. 
A possible explanation for the significant decrease in return visits is increased patient certainty in the final diagnosis. By being presented a highly accurate test result, the patient feels secure in the discharge instructions. This is important for seasonal and pandemic influenza planning, as it decreases the number of influenza-negative patients returning to the clinic. By returning to the clinic, they overwhelm the outpatient and emergency resources and put themselves at increased risk for influenza infection. ${ }^{14}$

Similarly, the reduction in antibiotic use may be due to increased clinician certainty in the diagnosis of influenza. By correctly identifying all or nearly all patients with influenza, fewer patients receive a noninfluenza diagnosis, resulting in fewer antibiotic prescriptions. This supports the idea that more accurate point of care diagnostics for influenza and other infections, such as Bordetella pertussis, Mycoplasma pneumonia, and Streptococcus pneumoniae, can be important tools in antibiotic stewardship efforts.

The need to adjust for clinic assignment has several possible explanations. The clinics contain different clinicians who may have different practice patterns and serve different proportions of the university student population. Patients in clinic 2 were less likely to receive guideline consistent care when adjusting for clinic assignment only (OR, 0.65; 95\% CI, 0.44-0.94). The composition of clinic staff may be important, as it has been previously demonstrated that nurse practitioners and physician assistants are more likely to prescribe an antibiotic for an acute respiratory infection compared to physicians. ${ }^{15}$

Interestingly, in some cases, clinicians did not accept or believe the PCR test results, despite the accuracy of the test. It is, therefore, important when introducing a new test to have clear instruction in the accuracy and interpretation of the test, including the positive and negative predictive value, as clinicians may have difficulty interpreting or understanding sensitivity and specificity.

Approximately 20 minutes was added to appointment times of patients who received the PCR test as compared to usual care. Many organizations make efforts to minimize the amount of time a patient spends in total at a primary care practice. Further research into the cost effectiveness of this test as the standard in primary care practice is needed. Students within our study did not pay for the rapid PCR test. The test currently reimburses at a maximum of $\$ 116.73$ nationally and $\$ 71.18$ in the state of Georgia. ${ }^{16}$ Comparatively, the rapid influenza tests cost between $\$ 12$ to 24 per test depending on brand. ${ }^{17}$ Although, from a health system perspective, the potential benefits include the reduction in antibiotic use, improved identification of patients with influenza resulting in a greater use of guideline consistent oseltamivir and earlier return to usual activities, and fewer return visits.

Our study had limitations. Most importantly, due to logistic constraints at the clinic, we were unable to randomize the enrollment of patients in our study. This resulted in baseline differences between patients in the PCR guided care group and the usual care group. The usual care group included all patients meeting our inclusion criteria, whereas patients in the PCR guided care group had to provide informed consent and could decline enrollment. Patients declining enrollment often told staff that they did not feel they were "sick enough" or were very certain "I do not have the flu." As a result, patients in the PCR guided care group had on average more symptoms and greater illness severity than those in usual care. Although we adjusted for this in our analysis, we may not have fully been able to account for unmeasured confounding. Second, not all our participants received a diagnostic test. Patients in usual care may have been treated empirically, meaning influenza diagnosis was never biologically confirmed.

\section{Conclusion}

In our study of PCR guided versus usual care for patients with ILI, patients who received PCR guided care were significantly less likely to receive an antibiotic, significantly more likely to receive oseltamivir, and were significantly less likely to have a return visit within 2 weeks. These are potential benefits of PCR guided care. However, given the quasiexperimental design, confirmation in a randomized trial is needed, as well a full evaluation of the cost-effectiveness of using the test.

To see this article online, please go to: http://jabfm.org/content/ 32/2/226.full.

\section{References}

1. Garten R, Blanton L, Elal AIA, et al. Update: influenza activity in the United States during the 2017-18 season and composition of the 2018-19 influenza 
vaccine. MMWR Morb Mortal Wkly Rep 2018;67: 634-42.

2. Fiore AE, Fry A, Shay D, Gubareva L, Bresee JS, Uyeki TM. Antiviral agents for the treatment and chemoprophylaxis of influenza-recommendations of the Advisory Committee on Immunization Practices (ACIP). MMWR Recomm Rep 2011;60:1-24.

3. Shiley KT, Lautenbach E, Lee I. The use of antimicrobial agents after diagnosis of viral respiratory tract infections in hospitalized adults: antibiotics or anxiolytics? Infect Control Hosp Epidemiol 2010;31: 1177-83.

4. Ebell MH, Radke T. Antibiotic use for viral acute respiratory tract infections remains common. Am J Manag Care 2015;21:e567-e575.

5. Steurer J, Held U, Spaar A, et al. A decision aid to rule out pneumonia and reduce unnecessary prescriptions of antibiotics in primary care patients with cough and fever. BMC Med 2011;9:56.

6. Biggs C, Walsh P, Overmyer CL, et al. Performance of influenza rapid antigen testing in influenza in emergency department patients. Emerg Med J 2010; 27:5-7.

7. Chartrand C, Leeflang MM, Minion J, Brewer T, Pai M. Accuracy of rapid influenza diagnostic tests: a meta-analysis. Ann Intern Med 2012;156:500-11.

8. Binnicker MJ, Espy MJ, Irish CL, Vetter EA. Direct detection of influenza $A$ and $B$ viruses in less than 20 minutes using a commercially available rapid PCR assay. J Clin Microbiol 2015;53:2353-4.

9. Gurol Y, Akan H, Izbirak G, et al. The sensitivity and the specifity of rapid antigen test in streptococcal upper respiratory tract infections. Int J Pediatr Otorhinolaryngol 2010;74:591-3.

10. FDA. Label: Oseltamivir Phosphate. 2016.

11. Harmes KM, Blackwood RA, Burrows HL, Cooke JM, Harrison RV, Passamani PP. Otitis media: diagnosis and treatment. Am Fam Physician 2013;88: 435-40.

12. Rosenfeld RM, Piccirillo JF, Chandrasekhar SS, et al. Clinical practice guideline (update): adult sinusitis. Otolaryngol Head Neck Surg 2015;152:S1-s39.

13. Burnham KP, Anderson DR. Multimodel inference: understanding AIC and BIC in model selection. Sociol Methods Res 2004;33:261-304.

14. Iuliano AD, Reed C, Guh A, et al. Notes from the field: outbreak of 2009 pandemic influenza A (H1N1) virus at a large public university in Delaware, April-May 2009. Clin Infect Dis 2009;49: 1811-20.

15. Sanchez GV, Hersh AL, Shapiro DJ, Cawley JF, Hicks LA. Outpatient antibiotic prescribing among united states nurse practitioners and physician assistants. Open Forum Infect Dis 2016;3:ofw168.

16. Centers for Medicare and Medicaid Services. Clinical lab fee schedule: Medicare and Medicaid. Available from: https://www.cms.gov/Medicare/Medicare-Feefor-Service-Payment/ClinicalLabFeeSched/. Published 2017.

17. Hueston WJ, Benich JJ. A cost-benefit analysis of testing for influenza A in high-risk adults. Ann Fam Med 2004;2:33-40. 